\title{
MEAN VALUES OF PERIODIC FUNCTIONS
}

\section{PAUL CIVIN}

Let $L^{p}$ denote the class of complex measurable functions of period $2 \pi$ for which $M_{p}(f)<\infty$, where

$$
\begin{aligned}
& M_{p}(f)=\left(\int_{0}^{2 \pi}|f(x)| p d x\right)^{1 / p} \quad(1 \leqq p<\infty), \\
& M_{\infty}(f)=\text { ess. } \sup _{0 \leqq x \leqq 2 \pi}|f(x)| .
\end{aligned}
$$

Let $K_{m, p}$ denote the subset of $L^{p}$ whose elements, $f(x)$, have a Fourier series of the form

$$
\sum_{n=m}^{\infty}\left(a_{n} \cos n x+b_{n} \sin n x\right) \quad(m \geqq 1) .
$$

The functions of $K_{m, p}$ and their Fourier series (3) are transformed by the real number $\delta$ and the sequence of real numbers $\lambda=\{\lambda(n)\}$ into the series

$$
\begin{aligned}
\sum_{n=m}^{\infty} \lambda(n) & \left\{a_{n} \cos \left(n x+\frac{\delta \pi}{2}\right)+b_{n} \sin \left(n x+\frac{\delta \pi}{2}\right)\right\} \\
=\sum_{n=m}^{\infty} \lambda(n) & \left\{\left(a_{n} \cos \frac{\delta \pi}{2}+b_{n} \sin \frac{\delta \pi}{2}\right) \cos n x\right. \\
+ & \left.\left(b_{n} \cos \frac{\delta \pi}{2}-a_{n} \sin \frac{\delta \pi}{2}\right) \sin n x\right\}
\end{aligned}
$$

A slight modification of the well known result ${ }^{1}\left[5, \mathrm{pp} .100 \mathrm{ff}^{2}\right]^{2}$ for the case in which $\delta=0$ shows that if

(5) $\sum_{n=m}^{\infty} \lambda(n) \cos \left(n x-\frac{\delta \pi}{2}\right)=\sum_{n=m}^{\infty} \lambda(n)\left\{\cos \frac{\delta \pi}{2} \cos n x+\sin \frac{\delta \pi}{2} \sin n x\right\}$

Presented to the Society, November 30,1946; received by the editors December $16,1946$.

${ }^{1}$ Although the convention is adopted in Trigonometrical series that $f(x)$ is real, the results of the sections of Trigonometrical series to which reference is made in this note hold for complex $f(x)$.

${ }^{2}$ Numbers in brackets refer to the bibliography at the end of the paper. 
is a Fourier or even a Fourier-Stieltjes series then (4) is the Fourier series of a function of $L^{p}, 1 \leqq p \leqq \infty$. Throughout the sequel it is assumed that (5) is a Fourier series. Series (4) is therefore the Fourier series of a function $g(x) \in L^{p}$ and is of the form of (3), hence $g(x) \in K_{m, p}$. The transformation determined by the number $\delta$ and the sequence $\lambda$ is thus a transformation of $K_{m, p}$ onto itself.

The objective of the present note is to establish an inequality between the means $M_{p}(f)$ and $M_{p}(g)$ which holds for all $f(x) \in K_{m, p}$. For the essentially bounded case this has been done by B. v. Sz. Nagy [3], and for completeness the result is stated as a lemma.

Lemma 1 [Sz. Nagy]. If $f(x) \in K_{m, \infty}$, then (4) is the Fourier series of a continuous function $g(x) \in K_{m, \infty}$ and

$$
M_{\infty}(g) \leqq A(\lambda, \delta, m) M_{\infty}(f),
$$

where $A(\lambda, \delta, m)$ is a function only of the indicated variables and not of the particular $f(x) \in K_{m, \infty}$.

The notation $A(\lambda, \delta, m)$ will be used throughout the sequel to denote the smallest possible function which will satisfy (6) for all $f(x) \in K_{m, \infty}$.

LEMMA 2. If $f(x) \in K_{m, 2}$, then (4) is the Fourier series of a function $g(x) \in K_{m, 2}$ and

$$
M_{2}(g) \leqq \Lambda(m) M_{2}(f),
$$

where $\Lambda(m)=\max _{m \leqq n}|\lambda(n)|$.

The Riesz-Fischer theorem asserts that (4) is the Fourier series of a function $g(x) \in L^{2}$ and that

$$
\begin{aligned}
M_{2}(g) & =\left\{\pi \sum_{n=m}^{\infty}(\lambda(n))^{2}\left(\left|a_{n}\right|^{2}+\left|b_{n}\right|^{2}\right)\right\}^{1 / 2} \\
& \leqq \Lambda(m)\left\{\pi \sum_{n=m}^{\infty}\left(\left|a_{n}\right|^{2}+\left|b_{n}\right|^{2}\right)\right\}^{1 / 2}=\Lambda(m) M_{2}(f) .
\end{aligned}
$$

If $\Lambda(m)=|\lambda(r)|(r \geqq m)$, then $f(x)=\cos r x$ gives equality in (7).

It is now possible to state the principal theorem.

THEOREM 1. If $f(x) \in K_{m, p}$, then (4) is the Fourier series of a function $g(x) \in K_{m, p}$ and

$$
\begin{array}{lr}
M_{p}(g) \leqq \Lambda^{2 / p}(m) A^{(p-2) / p}(\lambda, \delta, m) M_{p}(f) & (2 \leqq p<\infty), \\
M_{p}(g) \leqq B_{p} \Lambda^{2 / p^{\prime}}(m) A^{\left(p^{\prime}-2\right) / p^{\prime}}(\lambda,-\delta, m) M_{p}(f) & (1<p \leqq 2)
\end{array}
$$


where $\Lambda(m)$ and $A(\lambda, \delta, m)$ are defined in Lemmas 1 and $2, B_{p}$ is a constant depending only on $p$ and not on the particular $f(x) \in K_{m, p}$, and $p^{\prime}=p /(p-1)$.

The transformation of $f(x)$ into $g(x)$, or of series (3) into series (4), is a linear transformation of $K_{m, 2}$ onto itself, and also of $K_{m, \infty}$ onto itself. The direct application of an interpolation scheme for $L^{p}$ fails in the attempt to establish (8) since the space $K_{m, p}$ is a nondense linear subspace of $L^{p}$. However, the proof of the interpolation result for $L^{p}$ as given in Trigonometrical series [5, p. $198 \mathrm{ff}$.] carries through for the space $K_{m, p}$ on the basis of the following lemma.

LEMMA 3. The step functions of $K_{m, p}$ are dense in $K_{m, p}$ in the metric of $L^{p}(1<p<\infty)$.

The step functions of $K_{m, p}$ are those functions of $K_{m, p}$ which assume only a finite number of values and assume each of these values on a finite sum of intervals in $(0,2 \pi)$. Suppose $f(x) \in K_{m, p}$ and $\eta$ is a positive number. The density of the continuous functions of $L^{p}$ requires the existence of a continuous function $h(x) \sim c_{0} / 2$ $+\sum_{n=1}^{\infty}\left(c_{n} \cos n x+d_{n} \sin n x\right)$ such that $M_{p}(f-h)<\eta$ and $\left|c_{0}\right| / 2$ $+\sum_{n=1}^{m-1}\left(\left|c_{n}\right|+\left|d_{n}\right|\right)<\eta$. The function $k(x)=h(x)-c_{0} / 2$ $-\sum_{n=1}^{m-1}\left(c_{n} \cos n x+d_{n} \sin n x\right)$ is therefore a continuous function of class $K_{m, p}$ and $M_{p}(f-k) \leqq M_{p}(f-h)+\eta(2 \pi)^{1 / p}<8 \eta$. Hence the continuous functions of $K_{m, p}$ are dense in $K_{m, p}$. It is sufficient therefore to show that the continuous functions of $L^{p}$ can be approximated uniformly by step functions of $K_{m, p}$.

Consider first a continuous $f(x) \in K_{1, p}$. For any positive $\eta$, there is a step function $s(x)$ such that $|f(x)-s(x)| \leqq \eta$ for all $x$. If $c=(1 / 2 \pi) \int_{0}^{2 \pi} s(x) d x$, then since $\int_{0}^{2 \pi} f(x) d x=0,|c| \leqq(1 / 2 \pi) \int_{0}^{2 \pi} \mid s(x)$ $-f(x) \mid d x \leqq \eta$. The step function $t(x)=s(x)-c$ is therefore in $K_{1, p}$ and $|f(x)-t(x)| \leqq 2 \eta$.

Suppose next that it has been demonstrated that the continuous functions of $K_{r, p}$ can be uniformly approximated by step functions of $K_{r, p}$ for $1 \leqq r<m$. Since $K_{r, p} \supset K_{s, p}$ if $r<s$, any continuous function of $K_{m, p}$ can be uniformly approximated by step functions whose Fourier coefficients of order less than $(m-1)$ vanish. Hence if $f(x)$ is a continuous function of $K_{m, p}$ and $\eta$ is a positive number, there is a step function $s(x) \in K_{m-1, p}$ such that $|f(x)-s(x)|<\eta$ for all $x$. Suppose that $c=(1 / 4) \int_{0}^{2 \pi} s(x) \cos (m-1) x d x$ and $d=(1 / 4) \int_{0}^{2 \pi} s(x) \sin (m-1) x d x$. Since $f(x) \in K_{m, p}$, both $|c|<2 \eta$ and $|d|<2 \eta$. Suppose the function $t(x)=s(x)-c \operatorname{sgn} \cos (m-1) x-d \operatorname{sgn} \sin (m-1) x$, where sgn $u=0$ if $u=0$ and $\operatorname{sgn} u=u /|u|$ if $u \neq 0$. It can be shown by direct calculation that the step function $t(x) \in K_{m, p}$. Since $|f(x)-t(x)| \leqq|f(x)-s(x)|$ 
$+|c \operatorname{sgn} \cos (m-1) x|+|d \operatorname{sgn} \sin (m-1) x|<5 \eta$, the function $t(x)$ gives the desired uniform approximation.

In order to establish (9), it is first noted [5, p. 105] that since $g(x) \in L^{p}$,

$$
M_{p}(g)=\sup \left|\int_{0}^{2 \pi} g(x) \overline{h(x)} d x\right|
$$

with the supremum taken over all $h(x)$ for which $M_{p^{\prime}}(h) \leqq 1$. Hence if $\eta$ is a positive number there is an $h(x)$ for which

$$
M_{p^{\prime}}(h) \leqq 1
$$

and

$$
M_{p}(g)-\eta \leqq\left|\int_{0}^{2 \pi} g(x) \overline{h(x)} d x\right| .
$$

Suppose that $h(x) \sim r_{0} / 2+\sum_{n=1}^{\infty}\left(r_{n} \cos n x+s_{n} \sin n x\right)$ and that $h_{m}(x) \sim \sum_{n=m}^{\infty}\left(r_{n} \cos n x+s_{n} \sin n x\right)$. A double application of Parseval's relation for functions of $L^{p}$ and $L^{p^{\prime}}$ shows that

$$
\begin{aligned}
\int_{0}^{2 \pi} g(x) \overline{h(x)} d x=\pi \sum_{n=m}^{\infty} & \left\{\lambda(n)\left(a_{n} \cos \frac{\delta \pi}{2}+b_{n} \sin \frac{\delta \pi}{2}\right) \overline{r_{n}}\right. \\
& \left.+\lambda(n)\left(b_{n} \cos \frac{\delta \pi}{2}-a_{n} \sin \frac{\delta \pi}{2}\right) \overline{s_{n}}\right\} \\
= & \pi \sum_{n=m}^{\infty}\left\{\lambda(n)\left(\overline{r_{n}} \cos \frac{\delta \pi}{2}-\overline{s_{n}} \sin \frac{\delta \pi}{2}\right) a_{n}\right. \\
& \left.+\lambda(n)\left(\overline{s_{n}} \cos \frac{\delta \pi}{2}+\overline{r_{n}} \sin \frac{\delta \pi}{2}\right) b_{n}\right\} \\
= & \int_{0}^{2 \pi} \overline{H(x)} f(x) d x
\end{aligned}
$$

where

$$
\begin{aligned}
\boldsymbol{H}(x) \sim \sum_{n=m}^{\infty} \lambda(n)\left\{\left(r_{n} \cos \frac{\delta \pi}{2}-\right.\right. & \left.s_{n} \sin \frac{\delta \pi}{2}\right) \cos n x \\
& \left.\left.+\left(s_{n} \cos \frac{\delta \pi}{2}+r_{n} \sin \frac{\delta \pi}{2}\right) \sin n x\right)\right\} \\
= & \sum_{n=m}^{\infty} \lambda(n)\left\{r_{n} \cos \left(n x-\frac{\delta \pi}{2}\right)+s_{n} \sin \left(n x-\frac{\delta \pi}{2}\right)\right\} .
\end{aligned}
$$


Thus $H(x)$ is the transform of $h_{m}(x)$ which is obtained by use of the number $-\delta$ and the sequence $\lambda$. The application of Hölder's inequality followed by the use of (8) then shows that

$$
\begin{aligned}
\left|\int_{0}^{2 \pi} \overline{H(x)} f(x) d x\right| & \leqq M_{p^{\prime}}(H) M_{p}(f) \\
& \leqq M_{p}(f) \Lambda^{2 / p^{\prime}}(m) A^{\left(p^{\prime}-2\right) / p^{\prime}}(\lambda,-\delta, m) M_{p^{\prime}}\left(h_{m}\right) .
\end{aligned}
$$

A well known result of M. Riesz [1] implies that

$$
M_{p^{\prime}}\left(h_{m}\right) \leqq B_{p} M_{p^{\prime}}(h)
$$

where $B_{p}$ depends only on $p$ and not on the functions involved. The combination of formulas (10) through (14) then shows that

$$
M_{p}(g)-\eta \leqq B_{p} \Lambda^{2 / p^{\prime}}(m) A^{\left(p^{\prime}-2\right) / p^{\prime}}(\lambda,-\delta, m) M_{p}(f)
$$

and (9) follows since $\eta$ was arbitrary.

The result of Theorem 1 will now be applied to integrals of functions of $K_{m, p}$. It is convenient to use the definition of the integral of order $\alpha$ which is due to Weyl [4]. For any positive $\alpha$, for $f(x) \in K_{m, p}$ and with Fourier series (3), the integral of order $\alpha, f_{\alpha}(x)$, is defined as

$$
f_{\alpha}(x)=\sum_{n=m}^{\infty} \frac{1}{n^{\alpha}}\left\{a_{n} \cos \left(n x-\frac{\alpha \pi}{2}\right)+b_{n} \sin \left(n x-\frac{\alpha \pi}{2}\right)\right\} .
$$

Thus $f_{\alpha}(x)$ is the transform of $f(x)$ of the type of (4) with $\delta=-\alpha$ and the sequence $\{\lambda(n)\}=\left\{n^{-\alpha}\right\}$. Various results are known concerning the relationship between $M_{\infty}\left(f_{\alpha}\right)$ and $M_{\infty}(f)$, the most inclusive of which is that of Sz. Nagy [3] who shows that

$$
\begin{aligned}
A(\lambda, \delta, m) & =A\left(\left\{n^{-\alpha}\right\},-\alpha, m\right) \\
& \leqq\left(4 / \pi m^{\alpha}\right)\left\{\left|\cos \frac{\alpha \pi}{2}\right| \sum_{v=0}^{\infty}(-1)^{v}(2 v+1)^{-(1+\alpha)}\right. \\
& \leqq\left(4 / \pi m^{\alpha}\right) H(\alpha) .
\end{aligned}
$$

It can also be seen from [3] that

$$
A\left(\left\{n^{-\alpha}\right\}, \alpha, n\right) \leqq\left(4 / \pi m^{\alpha}\right) H(\alpha) .
$$

A direct application of Theorem 1 yields the following theorem.

THEOREM 2. If $f(x) \in K_{m, p}$, and $f_{\alpha}(x)$ is its integral of order $\alpha$ ( $\alpha$ not necessarily integral) then 


$$
\begin{array}{lr}
M_{p}\left(f_{\alpha}\right) \leqq m^{-\alpha}(4 H(\alpha) / \pi)^{(p-2) / p} M_{p}(f) & (2 \leqq p), \\
M_{p}\left(f_{\alpha}\right) \leqq B_{p} m^{-\alpha}(4 H(\alpha) / \pi)^{(2-p) / p} M_{p}(f) & (1<p \leqq 2)
\end{array}
$$

where $B_{p}$ is the constant of Theorem 1 and

$$
\begin{gathered}
H(\alpha)=\left|\cos \frac{\alpha \pi}{2}\right| \sum_{v=0}^{\infty}(-1)^{v}(2 v+1)^{-(1+\alpha)} \\
+\left|\sin \frac{\alpha \pi}{2}\right| \sum_{v=0}^{\infty}(2 v+1)^{-(1+\alpha)} .
\end{gathered}
$$

A result of Schmidt [2] shows that for the real functions of $K_{1, p}$ and $\alpha$ integral the coefficient of $M_{p}(f)$ in (15) is not the best possible.

\section{BIBLIOGRAPHY}

1. M. Riesz, Sur les fonctions conjuguées, Math. Zeit. vol. 27 (1928) pp. 218-244.

2. E. Schmidt, Über die Ungleichung, welche die Integral über ein Potenz einer Funktion und iber eine andere Potenz ihrer Ableitung verbindet, Math. Ann. vol. 117 (1940) pp. 301-326.

3. B. v. Sz. Nagy, Über gewisse Extremalfragen bei transformierten trigonometrischen Entwicklungen, I. Periodischer Fall, Saechsische Akademie der Wissenschaften, Mathematisch-physische Klasse, Berichte vol. 40 (1938).

4. H. Weyl, Bemerkung zum Begriff der Differentialquotienten gebrochener Ordnung, Naturforschende Gesellschaft, Zurich, Vierteljahrsschrift vol. 62 (1917) pp. 926-302.

5. A. Zygmund, Trigonometrical series, Warsaw-Lw6w, 1935.

UNIVERSITY OF OREGON 